\title{
The Composite of Bone Marrow Concentrate and Nano-hydroxyapatite as an Alternative to Autologous Bone in Reconstruction of Residual Surgical Defects
}

\author{
Mahmoud Abdel Aziz El Fadly*, Heba Abdel Wahed Selim, Salah Abdel Fattah Ahmed \\ Department of Oral and Maxillofacial Surgery, Faculty of Dentistry, Ain Shams University, Cairo, Egypt \\ Email address: \\ mahmoud_fadly@asfd.asu.edu.eg (M. A. A. El Fadly), tarek_elzayat1959@yahoo.com (H. A. W. Selim), \\ drsalahabdelfattah@yahoo.com (S. A. F. Ahmed) \\ ${ }^{*}$ Corresponding author

\section{To cite this article:} \\ Mahmoud Abdel Aziz El Fadly, Heba Abdel Wahed Selim, Salah Abdel Fattah Ahmed. The Composite of Bone Marrow Concentrate and \\ Nano-hydroxyapatite as an Alternative to Autologous Bone in Reconstruction of Residual Surgical Defects. International Journal of Clinical \\ Oral and Maxillofacial Surgery. Vol. 6, No. 2, 2020, pp. 20-25. doi: 10.11648/j.ijcoms.20200602.11
}

Received: August 5, 2019; Accepted: Sptember 9, 2019; Published: June 15, 2020

\begin{abstract}
The aim of this study was to examine the evidence behind the use of the composite of bone marrow concentrate and Nano-hydroxyapatite as an alternative to autologous bone graft in the repair of bony defects. The study was conducted on 14 residual surgical non-continuity defects resulting from enucleation of benign odontogenic cysts. The defects were randomly allocated into two groups according to defect filling as follows: Group A ( $n=7)$, the defects were grafted using a composite of bone marrow concentrate (BMC) and Nano-hydroxyapatite granules; Group B ( $=7)$, the defects were grafted using autologous bone graft. Radiographic evaluation was done using Cone beam C. T. scan, immediate, six and twelve months post-operatively to assess the volumetric changes of the grafts along the follow-up period. The results of this study revealed that there was no statistically significant difference $(p>0.05)$ between the two groups regarding the percentage of reduction of the graft volume along the follow-up period. In the light of the radiographic results of this study it can be concluded that the use of composite of bone marrow concentrate (BMC) and Nano-hydroxyapatite granules to reconstruct surgical defects is a reliable alternative to autogenous bone graft.
\end{abstract}

Keywords: Bone Marrow Concentrate, Autogenous Bone Graft, Nano-hydroxyapatite, Surgical Defects

\section{Introduction}

Bone tissue usually heals spontaneously, but in complicated conditions such as those situations leading to large bony defects or pathological fractures, spontaneous healing process may fail. Therefore, surgical reconstruction of bony defects is still a challenge for surgeons to rehabilitate patients with severe bone deformities to restore continuity, shape and strength of the jaw to achieve better patient's quality of life [1].

Autogenous bone graft has been repeatedly reported to be the gold standard, however, complications related to the harvest of autografts as donor site morbidity and limited availability demanded the search for an alternative with a comparable efficiency [2-5].

With the widespread use of cellular therapy in medical practice, attention has been directed to the application of autologous bone marrow in the field of reconstruction. Bone marrow aspirate (BMA) has been utilized as a source of bone marrow-derived mesenchymal stem cells (MSCs) with its relative ease of harvest, low morbidity, and feasible cost. BMA alone has a relatively low percentage of MSCs. Hence, the aspirate is typically concentrated by centrifugation so as to increase the ratio of MSCs. Concentrated bone marrow aspirate (BMC) provides both stem cells and growth factors and relies on the host tissue to provide scaffold [6-9].

The available literature regarding the use of BMC in different tissue repair is highly heterogeneous with regards to indications, concentrations and overall functional 
outcomes. Hence, this study attempts to examine the evidence behind the use of BMC as an alternative to autologous bone graft in the repair of bony defects and to establish proof of concept for the use of BMC in this biologic environment.

\section{Methodology}

The clinical study was conducted on 14 residual surgical non-continuity defects resulting from enucleation of benign odontogenic cysts with a low recurrence rate.

The cases were recruited from patients attending the outpatient clinic of the Oral and Maxillofacial surgery department, Faculty of Dentistry, Ain Shams University.

The study was conducted after receiving an ethical clearance from the Research Ethics Committee of Ain Shams University, Faculty of Dentistry; that the study follows the guideline of research ethical committee.

\subsection{Patient Selection}

The patients were included into the study based on the following inclusion criteria:

1) Male and female patients with minimum age 18 years old and maximum age 50 years old.

2) Medically free from systemic diseases.

3) Patients with mandibular/maxillary two or three walls non- discontinuity residual surgical defects, providing that the palatal/lingual wall is intact, resulting from enucleation of benign odontogenic cyst with low recurrence rate.

4) Non-smoker patients.

5) Absence of local conditions that may affect bone healing.

\subsection{Patient Grouping}

Patients were divided into two groups. Allocation of patients included in this study were done by coin toss by noninvolved investigator.

1) Group A (study group): included seven residual surgical non-continuity defects where grafting of the defects was done using a composite of bone marrow aspirate concentrate (BMAC) and Nano-hydroxyapatite particles.

2) Group B (control group): included seven residual surgical non-continuity defects where grafting of the residual surgical defects was done using autologous bone graft harvested from anterior iliac crest.

\subsection{Surgical Procedure in Group A Patients (Study Group)}

\subsubsection{Recipient Site Preparation}

General anesthesia was induced then a three- line pyramidal muco-periosteal flap was designed according to the extent of the lesion. Enucleation and curettage of the lesion was done using bone curettes. Horizontal scoring of the periosteum was performed to guarantee tissue flexibility during flap closure.

\subsubsection{Residual Defect Grafting Procedure}

The residual surgical non-continuity defects were grafted using Nano- hydroxyapatite granules (Nano Bone $\AA$, ARTOSS GmbH, Germany) $1 \mathrm{~mm}$ particle size. The graft granules were packed tightly to ensure complete filling of the surgical defect. (Figure 1).

The flap was then repositioned using interdental 4-0 Braided Polyglycolic Acid sutures, vertical releasing incisions were closed using interrupted 4-0 Braided Polyglycolic Acid sutures.

\subsubsection{Bone Marrow Aspiration Procedure}

Patients in group A (study group) were prepared for harvesting bone marrow aspirate from an anterior iliac crest approach using a trocar needle (Figure 2). In this study, 60 $\mathrm{ml}$ of bone marrow was aspirated from each patient in the study group. The aspirated bone marrow was collected on heparinized tubes and refrigerated until it was concentrated in a later step.

\subsubsection{Preparation of Bone Marrow Aspirate Concentration (BMAC)}

This procedure was performed in Pancreatic Cell Culture and Diabetes Research Unit, Faculty of Medicine, Ain Shams University. The harvested bone marrow sample was treated by Ficoll protocol [10] to prepare bone marrow concentrate according to the following steps:

1) The harvested bone marrow was diluted by Dulbecco's modified eagle's medium (DMEM) (Biowhittaker ${ }^{\circledR}$ Lonza, Switzerland) in ratio 4:1.

2) Bone marrow was then centrifuged at $2000 \mathrm{rpm}$ for 20 minutes at $20^{\circ} \mathrm{C}$ in a swinging-bucket rotor without brake.

3) Aspiration of the mononuclear cells rich layer using micro-pipette was done to be re-diluted by DMEM and centrifuged again at $1200 \mathrm{rpm}$ for 10 minutes at $20^{\circ} \mathrm{C}$ and this was repeated twice to ensure high concentration of mononuclear mesenchymal stem cells.

4) Streptomycin was then added to the prepared bone marrow aspirate concentrate for prophylaxis against infection. The $\mathrm{BMC}$ was then gradually frozen to $30^{\circ} \mathrm{C}$ then $-70^{\circ} \mathrm{C}$ before it was stored at $-200^{\circ} \mathrm{C}$ using liquid nitrogen.

\subsubsection{Application of Bone Marrow Aspirate Concentrate in Group A Patients}

An appointment had been scheduled for patients in group A (study group) one week post-operative to inject the previously prepared bone marrow aspirate concentrate (BMAC) into the grafted site according to the following steps.

1) Local anesthesia had been administrated using Mepivacaine (2\%), levonordefrin (1:20000).

2) 2-3 $\mathrm{ml}$ of BMAC was then injected into the grafted site using plastic disposable syringe (18 gauge needle). (Figure 3) 


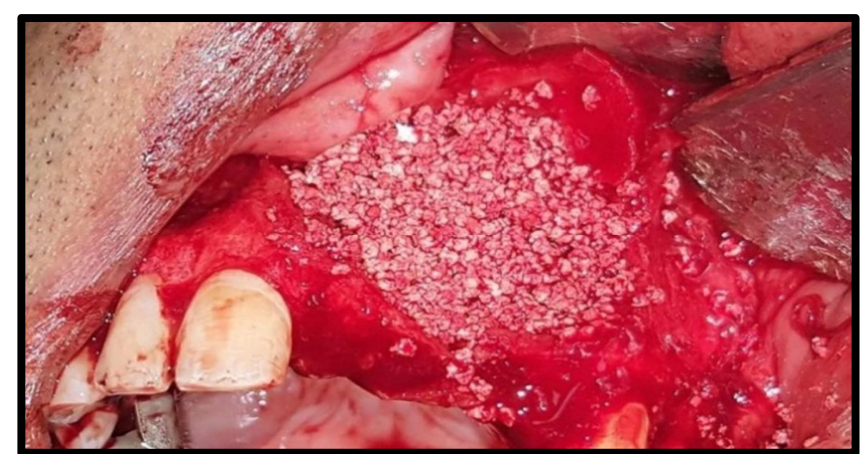

Figure 1. Complete filing of residual surgical defect in group A using Nanohydroxyapatite granules.

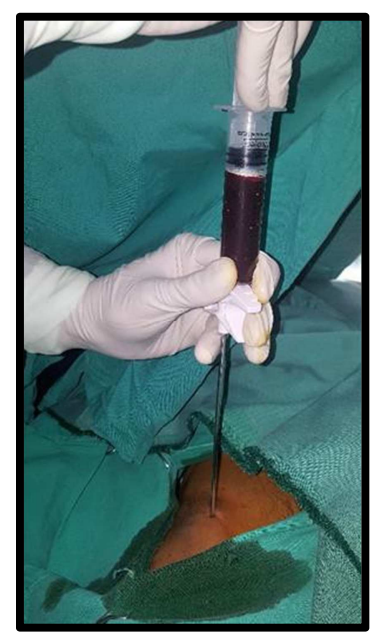

Figure 2. Bone marrow aspiration procedure in group A.

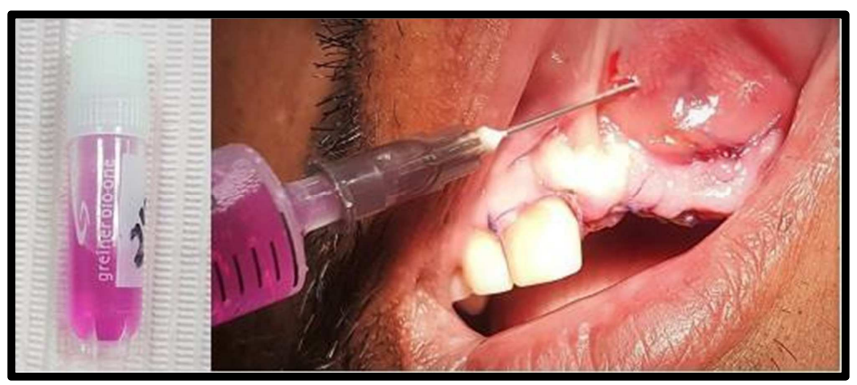

Figure 3. Injection of bone marrow aspirate concentrate into the grafted site in group $A$.

\subsection{Surgical Procedure in Group B Patients (Control Group)}

\subsubsection{Recipient Site Preparation}

General anesthesia was induced then the recipient site was prepared the same way as in the study group.

\subsubsection{Donor Site Preparation}

The patients in group B (control group) were prepared for harvesting the anterior iliac crest bone graft. The harvested bone particulate was kept in a kidney dish filled with normal saline and gentamicin $40 \mathrm{mg}$.

\subsubsection{Residual Defect Grafting Procedure}

The residual surgical non-continuity defects were grafted using the harvested anterior iliac crest bone graft. Harvested cancellous bone was collected in plastic syringe to calculate the total volume of bone then the graft granules were packed tightly to ensure complete filling of the surgical defect. The flap was then repositioned using interdental 4-0 Braided Polyglycolic Acid sutures, vertical releasing incisions were closed using interrupted 4-0 Braided Polyglycolic Acid sutures.

\subsection{Method of Evaluation}

Radiographic evaluation using Cone beam C. T. scan was done at the following intervals:

1. Immediate postoperatively.

2. 6 and 12 months postoperatively.

After CBCT imaging, all data were reconstructed with a 1 $\mathrm{mm}$ slice thickness and $1 \mathrm{~mm}$ interval. These scans were converted into (DICOM) format files (NNT software v. 4.6) and imported into software Mimics 19.0†, where they could be viewed and assessed using the various tools available by this program.

For volumetric calculation of the bone graft material, window level and window width were adjusted to clearly extract the border between the graft and normal bone. Tracing was achieved by free hand method on axial and sagittal slices as well depending on observer's visual skills to delineate the bone graft after thresholding (adjusting density).

\subsection{Data Management and Analysis}

The collected data was revised, coded, tabulated and introduced to a personal computer using Statistical package for Social Science (IBM Corp. Released 2013. IBM SPSS Statistics for Windows, Version 22. Armonk, NY: IBM Corp).

1) Paired-Samples $T$ Test was used to assess the statistical significance of the difference between two means of one quantitative variable measured twice for the same study group. Namely, the volumetric assessment immediately post-operative compared to that measured at six months and at twelve months within each group.

2) The Independent-Samples T Test was used to assess the statistical significance of the difference between the mean of change in bone volume of group A compared to those of group B both at the six and twelve months' intervals as well as the mean of change in bone volume in both groups.

\section{Results}

Single oral radiologist, evaluated all CBCT data sets. These data were re- assessed separately after four weeks following the first evaluation under the same settings by the same oral radiologist to determine the intra-observer error. Separate spreadsheets were used to record the first and the second measurements to avoid analytical bias from the first results. The concordance correlation coefficient statistical test revealed substantial strength of agreements $(p=0.95-0.99)$ between the two measurements 


\subsection{Description of Post-operative Radiographs}

Radiographic examination revealed that there were gradual reduction in size of bone defect in all cases during follow up period. Cone beam C. T. scan obtained 6 months postoperative revealed regenerated bone architecture excluding central portion of surgical site. Cone beam C. T. scan obtained one year post-operative revealed almost complete bone healing manifested by formation of normal trabecular pattern (Figure 4).

\subsection{Assessment of Volumetric Changes in Graft Volume}

Assessment of change in graft volume in this study showed that, among the control group, graft volume decreased significantly at six months and one year postoperative when compared to immediate post-operative volume. Also, among the study group, graft volume decreased significantly at six months and one year postoperative when compared to immediate post- operative volume (figure 5). However, there was no statistically significant difference between control and study groups regarding percentage of reduction in graft volume along follow-up period.
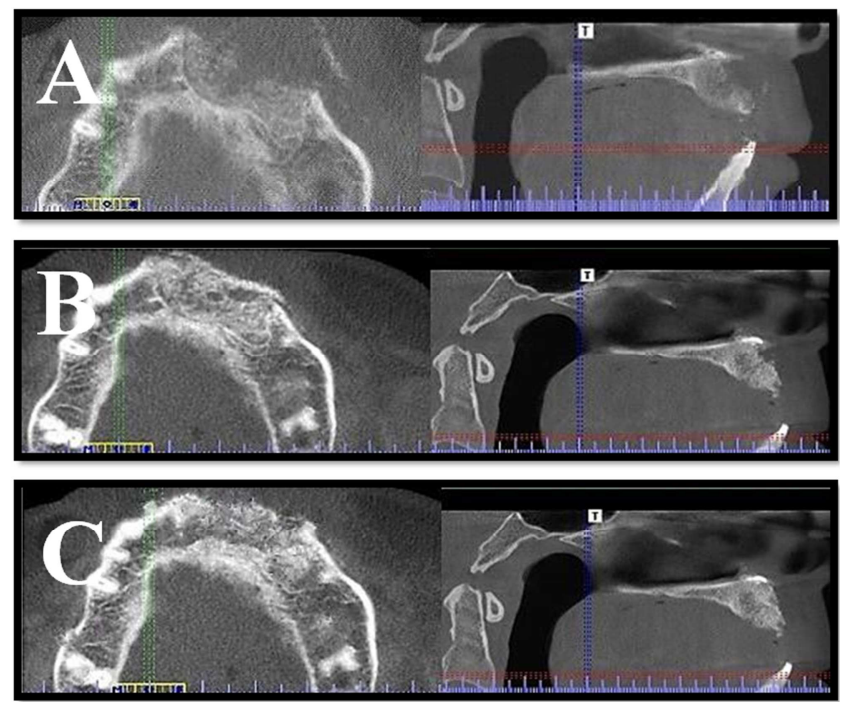

Figure 4. Postoperative CBCT scan (axial and sagittal cuts) for residual surgical defect reconstructed BMC + Nano-hydroxyapatite granules. A. immediate postoperative, $B$. six months postoperative, $C$ one year postoperative.

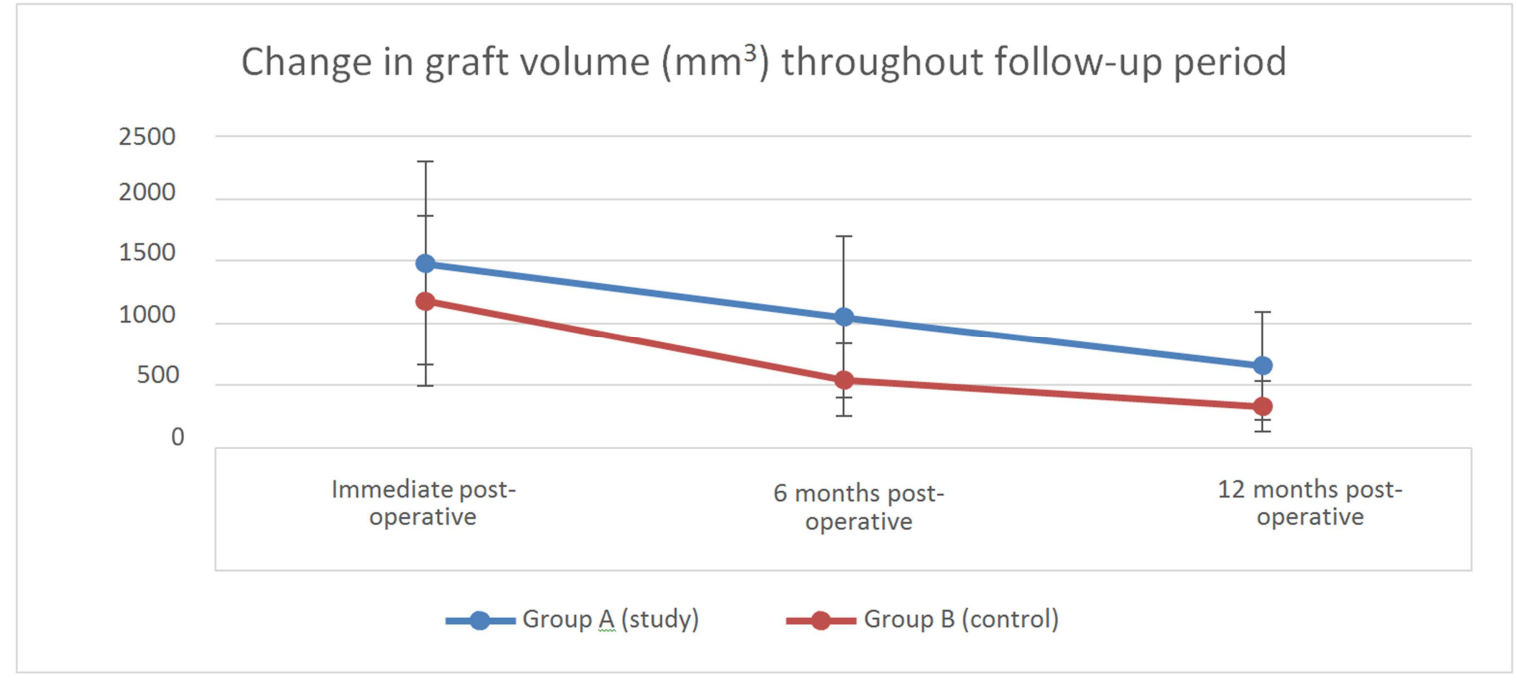

Figure 5. Change in graft volume among control and study groups along follow up period.

\section{Discussion}

Reconstruction of residual surgical defects resulting from enucleation of benign odontogenic cysts is a debatable issue in the literature. Although several studies advocated primary closure without the use of additional bone grafts, grafting of surgical defects is recommended in defects with more than one cortical border missing or in defects with incomplete periosteal covering as it may prevent collapse of the surrounding soft tissue, preserve the defect volume, and provide osteogenic cells and growth factors for regular bone healing [11].

Autogenous bone grafts have long been considered as the gold standard for reconstruction of residual surgical defects as it possesses the three classic qualities of the ideal graft, including osteo-induction, osteo-conduction, and osteogenesis. In addition, they have complete histocompatibility, thus generating a minimum immunological reaction. However, their harvesting is associated with donor site morbidity and they have a restricted availability. Therefore, the search for an alternative with an osteogeneic potential equivalent to autogenous bone grafts is mandatory.

At an increasing rate cell therapeutics, such as mesenchymal stem cells from bone marrow in combination with osteoconductive bone substitutes are being used today [12-14]. One possible application method of cell therapeutics is the use of bone marrow concentrate (BMC), which is obtained by density gradient centrifugation $[6,7]$. So the aim of this study was to find out whether this modality could possibly be a substitute for autogenous bone graft or not. 
Ficoll density gradient centrifugation was the protocol adopted to concentrate bone marrow in this study, being one of the most common methods for obtaining bone marrow stem cells with acceptable purity, viability, and cost $[10,15$, $16]$.

The age inclusion criteria in the current study was restricted to the patient below 50 years old as a result of the fact of sequential deterioration of osteogenic potential of osteoblasts after age of 50 [17].

Residual surgical defects included in this study resulted from enucleation of benign odontogenic cysts with low recurrence rate which is an appropriate condition for primary grafting. Since grafting of the residual surgical defects was done using particulate cancellous bone in the control group and the composite of BMC and Nano-hydroxyapatite granules in the study group so the morphology of the residual surgical defects included in the study was chosen to be two or three walls non-discontinuity defects, providing that the palatal/lingual wall was intact in order to contain the graft material.

For the most common Ficoll method, an important limitation is the time required for the extended laboratory steps necessary for concentrating bone marrow which have made preparation of the bone marrow concentrate intraoperative practically inconvenient. For this reason, application of bone marrow concentrate was scheduled one week post-operative instead of mixing BMC with Nanohydroxyapatite granules intra-operative.

The delayed application of bone marrow concentrate can be also justified by the fact that the first week following cyst enucleation corresponds to the initial phase of healing at which application of bone marrow concentrate may be beneficial to bone healing. This suggestion was in agreement with a previous study that introduced bone marrow concentrate to a distracted mandibular segment after ten and twenty days of consolidation aiming at improving bone healing and maturation [18].

A crucial aspect to achieve successful bone regeneration using bone substitutes is the resorption of the bone substitute with simultaneous formation of new bone. In this study, the degradation of the graft and whether it was coupled with shrinkage in defect volume or not was assessed using cone beam C. T. scan. The superiority of CBCT to plain radiography is now undebatable [19].

Postoperative CBCT was performed immediately, at six and twelve months which are repeatedly reported time intervals suitable for assessment of bone healing in humans. The data from CBCT images was assessed by Mimics Ver. 19 software for volumetric assessment. This allowed better evaluation of the graft and the defect volume postoperatively through segmentation of the interested area to measure its volume separately $[20,21]$.

The immediate post-operative CBCT was justified by the need to establish a baseline in order to assess reduction in graft and defect volume at 6 and 12 months as the grafted bone and the defect margin were clearly distinguished from the surrounding cortical bone.
While traditional hydroxyapatite is generally considered as nonresorbable, resorbability is supposed to occur for Nanosized hydroxyapatite based on the greater solubility [22]. However, several studies reported that Nano-hydroxyapatite granules when used for filling of surgical defects had shown incomplete degradation with fibrous encapsulation of the residual particles which may hinder complete ossification of the bony defect [11].

In contrast, in the current study, among the study group, residual surgical defect volume decreased significantly at six months and one year post-operative when compared to immediate post-operative volume indicating proper substitution of the graft granules with new bone trabeculae along the follow up period.

The percentage of reduction of graft volume in the study group was $29.13 \% \pm 11.53$ at six months post-operative. In a recent study which assessed volumetric shrinkage of Nanohydroxyapatite granules used in augmentation after sinus membrane elevation procedure, the percentage of reduction of graft volume at six months post-operative was $24.12 \%$ [23].

When comparing the previously mentioned results, it may be suggested that bone marrow concentrate may have accelerated degradation of the Nano-hydroxyapatite granules which allows proper replacement of the granules by new bone formation. Increased rate of graft resorption also can be due to the effect of silica ions incorporated in the structure of Nano-bone that may activate the differentiation of osteogenic precursor cells into osteoclasts. However, the difference in the method of assessment of volumetric change should be put into consideration when comparing the results.

When comparing the percentage of graft reduction till six months and one year post-operative between the control and study groups, there was no statistical significant difference between the two groups. This suggests that bone marrow concentrate may have potentiated the biological activity of Nano-hydroxyapatite granules. Accordingly, it can be postulated that the composite of BMC and Nanohydroxyapatite may be a reliable substitute for autogenous bone graft.

\section{Conclusion}

In the light of the radiographic and histological results of this study, the application of the composite of BMC +Nanohydroxyapatite could be a comparable alternative to autologous bone suitable for reconstruction of residual surgical defects thus minimizing donor site morbidity and achieving patient satisfaction.

\section{Funding}

Authors' own fund.

\section{Conflicts of Interest}

The authors declare that they have no competing interests. 


\section{Acknowledgements}

Not applicable.

\section{References}

[1] Packer JW, Colditz JC. Bone injuries: treatment and rehabilitation. Hand Clin. 1986; 2: 81-91.

[2] Hall HD, Phillips RM, Chase DC. Bone grafts of large cystic defects in the mandible. J Oral Surg. 1971; 29: 146-150.

[3] Giannoudis PV, Dinopoulos H, Tsiridis E. Bone substitutes: an update. Injury. 2005; 36: S20-S27.

[4] Pradel W, Eckelt U, Lauer G. Bone regeneration after enucleation of mandibular cysts: comparing autogenous grafts from tissue- engineered bone and iliac bone. Oral Surg Oral Med Oral Pathol Oral Radiol Endod. 2006; 101: 285-290.

[5] Younger EM, Chapman MW. Morbidity at bone graft donor sites. J Orthop Trauma. 1989; 3: 192-195.

[6] Jager M, Hernigou P, Zilkens C, Herten M, Fischer J, et al. Cell therapy in bone-healing disorders. Orthopade. 2010; 39: 449-462; quiz 463.

[7] Rosset P, Deschaseaux F, Layrolle P. Cell therapy for bone repair Orthopaedics \& traumatology, surgery \& research: OTSR 2014; 100: S107-S112.

[8] P. Kasten, I. Beyen, M. Instant stem cell therapy: characterization and concentration of human mesenchymal stem cells in vitro. European Cells \& Materials, vol. 16. 2008; $47-55$.

[9] Chahla J, Mannava S, Cinque ME, Geeslin AG, Codina D, LaPrade RF. Bone Marrow Aspirate Concentrate Harvesting and Processing Technique. Arthrosc Tech. 2017; 6: 441-45.

[10] Al Battah F, De Kock J, Ramboer E, Heymans A, Vanhaecke T. Evaluation of the multipotent character of human adipose tissue- derived stem cells isolated by Ficoll gradient centrifugation and red blood cell lysis treatment. Toxicol in Vitro. 2011; 25: 1224-1230.

[11] Ettl T, Gosau M, Sader R, Reichert TE. Jaw cysts - filling or no filling after enucleation? A review. J Craniomaxillofac Surg. 2012; 40: 485-93.

[12] Kitoh H, Kitakoji T, Tsuchiya H, Katoh M, Ishiguro N Transplantation of culture expanded bone marrow cells and platelet rich plasma in distraction osteogenesis of the long bones. Bone 2007: 40: 522-528.
[13] Jager M, Herten M, Fochtmann U, Fischer J, Hernigou P. Bridging the gap: bone marrow aspiration concentrate reduces autologous bone grafting in osseous defects. J Orthop Res 2011; 29: 173-180.

[14] Hatzokos I, Stavridis SI, Iosifidou E, Karataglis D, Christodoulou A. Autologous bone marrow grafting combined with demineralized bone matrix improves consolidation of docking site after distraction osteogenesis. J Bone Joint Surg Am 2011; 93: 671-678.

[15] Peterbauer-Scherb A, van Griensven M, Meinl A, Gabriel C, Redl H. Isolation of pig bone marrow mesenchymal stem cells suitable for one-step procedures in chondrogenic regeneration. J Tissue Eng Regen Med. 2010; 4: 485-490.

[16] Horn P, Bork S, Diehlmann A, Walenda T, Eckstein V. Isolation of human mesenchymal stromal cells is more efficient by red blood cell lysis. Cytotherapy. 2008; 10: 676-685.

[17] Marx RE, Garg AK. Bone structure, metabolism, and physiology: its impact on dental implantology. Implant Dent 1998; 7: 267-276.

[18] El Hadidi YN, El Kassaby M, El Fatah Ahmed SA, Khamis NS. Effect of Mesenchymal Stem Cell Application on the Distracted Bone Microstructure: An Experimental Study. J Oral Maxillofac Surg. 2016; 74: 1463. e1-1463. e11.

[19] Rosenstein SW, Long Jr RE, Dado DV, Vinson B, Alder ME. Comparison of 2-D calculations from periapical and occlusal radiographs versus 3-D calculations from CAT scans in determining bone support for cleft-adjacent teeth following early alveolar bone grafts. The Cleft palate-craniofacial journal. 1997; 34: 199-205.

[20] Zhang W, Shen G, Wang X, Yu H, Fan L. Evaluation of alveolar bone grafting using limited cone beam computed tomography. Oral Surg Oral Med Oral Pathol Oral Radiol. 2012; 113: 542-8.

[21] El Badawy F, Hamed W, Farid M, El Kassaby M. Volumetric Analysis of Alveolar Bone Grafts using Cone Beam Computed Tomography. Egypt Dent J. 2015; 62 (3).

[22] Jensen SS, Bornstein MM, Dard M, Bosshardt DD, Buser D: Comparative study of biphasic calcium phosphates with different HA/TCP ratios in mandibular bone defects. A longterm histomorphometric study in minipigs. J Biomed Mater Res B Appl Biomater. 2009; 90: 171e181.

[23] Lorenz J, Eichler K, Barbeck M. Volumetric analysis of bone substitute material performance within the human sinus cavity of former head and neck cancer patients: A prospective, randomized clinical trial. Ann Maxillofac Surg. 2016; 6: 175-181. 